\title{
Cardiac Motion and Elasticity Characterization with Iterative Sequential $\mathcal{H}_{\infty}$ Criteria
}

\author{
Huafeng Liu ${ }^{1,2}$ and Pengcheng Shi ${ }^{2}$ \\ 1 State Key Laboratory of Modern Optical Instrumentation \\ Zhejiang University, Hangzhou, China \\ 2 Department of Electrical and Electronic Engineering \\ Hong Kong University of Science and Technology, Hong Kong \\ \{eeliuhf, eeship\}@ust.hk
}

\begin{abstract}
Robustness is of paramount importance in cardiac wall motion estimation and myocardial tissue elasticity characterization, especially for clinical applications. Given partial, noisy, image-derived measurements on the cardiac kinematics, we present an integrated robust estimation framework for the joint recovery of dense field cardiac motion and material parameters using iterative sequential $\mathcal{H}_{\infty}$ criteria. This strategy is particulary powerful for real-world problems where the types and levels of model uncertainties and data disturbances are not available a priori. Constructing the myocardial dynamics equations from biomechanics principles, at each time step, we rely on techniques from $\mathcal{H}_{\infty}$ filtering theory to first generate estimates of heart kinematics with suboptimal material parameter estimates, and then recover the elasticity property given these kinematic state estimates. These coupled iterative steps are repeated as necessary until convergence. We demonstrate the accuracy and robustness of the strategy through experiments with both synthetic data of varying noises and magnetic resonance image sequences.
\end{abstract}

\section{Introduction}

Regional wall function and myocardial tissue properties reveal critical information about the states of cardiac physiology and pathology. With increasingly available real-time and ECG-gated tomographic cardiac imaging data, there have been plenty image-based efforts aimed at measuring the motion and/or the material properties of the heart [3.9].

Given a set of image-derived, sparse, noisy measurements on cardiac kinematics, typical motion analysis methods need to make use of additional constraining models of mathematical or mechanical nature to obtain the dense field motion fields in some optimal senses. The underlying hypothesis is, of course, that these prior models are completely known, including their parameters, and are appropriate for the particular image data. For practical situations, especially those pathological cases, however, this assumption is rarely true. Some of the related frame-to-frame strategies can be found in a comprehensive recent review [3]. Assuming Gaussian conditions on system and data uncertainties, there are several 

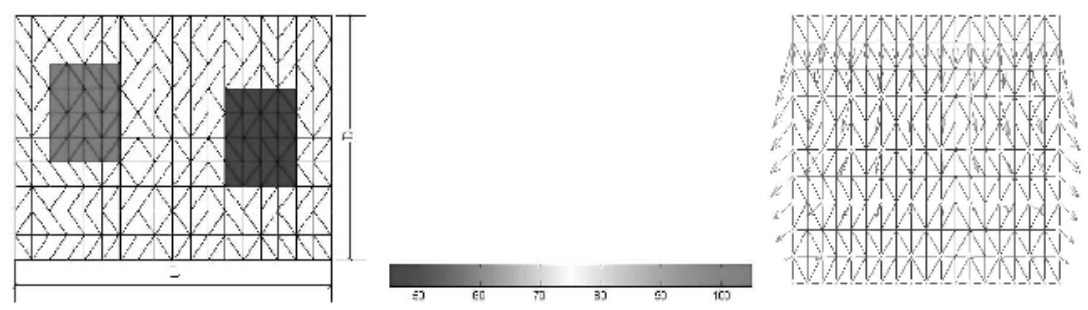

Fig. 1. Generation of the synthetic kinematic data: original configuration (left), color scale of material elasticity (middle), and displacement data at selected locations (right).

multi-frame efforts which impose the all important spatio-temporal constraints to perform motion analysis throughout the cardiac cycle [5]814].

The dual problem to cardiac motion recovery is the estimation of myocardial tissue elasticity based on known, image-derived kinematics observations. There have been a number of efforts on the parameter identification of the myocardium, where the basic idea is to minimize some criteria that measure the goodness of fit between the model-predicted and the data-derived mechanical responses 19. More recently, an expectation-maximization (EM) strategy is proposed to estimate the composite tissue mechanical properties by using displacements reconstructed from MR tagging data [4].

We have shown that it is desirable to tackle the motion and elasticity estimation problems simultaneously, especially for disease conditions where the myocardial tissue structure and parameters undergo substantial alterations 6] 11. By making use of the stochastic finite element and the extended Kalman filter [11, followed by a Bayesian scheme based on the maximum a posteriori formulation [6], these efforts were built upon a biomechanical model of the myocardium and were embedded within $\mathcal{H}_{2}$ filtering frameworks under known Gaussian statistics assumptions, unrealistic for real world problems. In order to relax these restrictions, we have recently proposed several $\mathcal{H}_{\infty}$-based robust strategies for the image-based motion recovery with fixed material model [7] and tissue elasticity estimation from known kinematics measurements [12].

In this paper, we present a robust $\mathcal{H}_{\infty}$ framework for the joint estimation of cardiac kinematics and material parameters from imaging data. It differs from the $\mathcal{H}_{2}$-based simultaneous estimation in: 1 ). no a priori knowledge of noise statistics is required; and 2). the min-max estimation criterion is to minimize the worst possible effects of the disturbances (model and data noises) on the signal estimation errors, which will ensure that if the disturbances are small (in energy), the estimation errors will be as small as possible (in energy), regardless the noise types. These two aspects make the $\mathcal{H}_{\infty}$ to be more appropriate for certain practical problems where the disturbances are unknown and nonGaussian. In addition, our strategy is posed as an iterative sequential estimation framework which uses two separated $\mathcal{H}_{\infty}$ filters: one for kinematics estimation (the $\mathcal{H}_{\infty}$ state-filter) and one for tissue elasticity estimation (the $\mathcal{H}_{\infty}$ parameterfilter). At every time step, the current estimates of material parameters are used in the state-filter, and the current estimates of the kinematics are used in the 

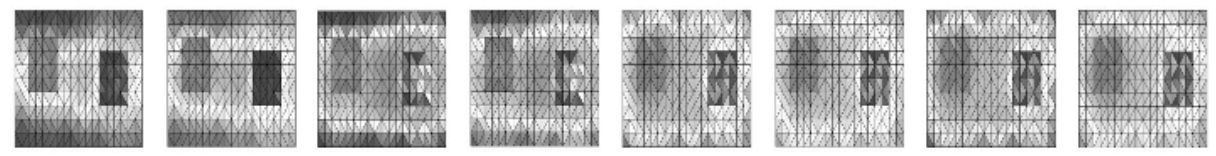

Fig. 2. Estimated elasticity distributions using the EKF [11] (the four figures on the left) and $\mathcal{H}_{\infty}$ (the four figures on the right) methods for noisy input data (left to right): $S N R=20 d B$ (Gaussian), $S N R=30 d B$ (Gaussian), $S N R=20 d B$ (Poisson), and $S N R=30 d B$ (Poisson).

parameter-filter. It can be thought as a generalization of the expectation maximization (EM) scheme, without the needs to know the underlying distributions.

\section{Methodology}

\subsection{System Dynamics from Continuum Mechanics Model}

For computational feasibility, our current implementation uses linear elastic material model for the myocardium. The myocardial dynamics equation, in terms of displacement field $U$, with a finite element representation is in the form of:

$$
M \ddot{U}+C \dot{U}+K U=R
$$

where $M$ is the mass matrix and $R$ the external load. The stiffness matrix $K$ is related to the material-specific Young's modulus $E$ and Poisson's ratio $\nu$. In this paper, $\nu$ is fixed to 0.49 for almost incompressible material and $E$ needs to be estimated along with the kinematics function 1 . We also assume Rayleigh damping with $C=\alpha M+\beta K$ with fixed weighting factors $\alpha$ and $\beta$.

\subsection{Motion Estimation: $\mathcal{H}_{\infty}$ State Filter}

Kinematics State Space Representation. In state-space representation, Equation (1) can be rearranged by making $x(t)=(U(t), \dot{U}(t))^{T}(T$ denotes transpose):

$$
\dot{x}(t)=A_{c}(\theta) x(t)+B_{c} w(t)
$$

where the material parameter vector $\theta$, the system matrices $A_{c}$ and $B_{c}$, and the input term $w$ are:

$$
\theta=E, \quad A_{c}=\left[\begin{array}{cc}
0 & I \\
-M^{-1} K & -M^{-1} C
\end{array}\right], \quad B_{c}=\left[\begin{array}{cc}
0 & 0 \\
0 & M^{-1}
\end{array}\right], \quad w(t)=\left[\begin{array}{l}
0 \\
R
\end{array}\right]
$$

For discrete system, assuming that the input is piecewise constant over the sampling interval $T$ between image frames, and adding the process noise $v(t)$, we arrive at the following system equation [1]:

$$
x(t+1)=A(\theta) x(t)+B(\theta) w(t)+v(t)
$$

with $A=e^{A_{c} T}$ and $B=A_{c}^{-1}\left(e^{A_{c} T}-I\right) B_{c}$.

\footnotetext{
${ }^{1}$ Here, we assume that $E$ is spatially varying but temporally constant.
} 
Table 1. EKF 11 and $\mathcal{H}_{\infty}$ estimated Young's moduli using the noise-corrupted synthetic data: each data cell represents the mean \pm standard derivation for the normal (75), hard (105), and soft (45) tissues.

\begin{tabular}{|c|c|c|c|c|c|}
\hline \hline Method & Tissue Type & 20dB(Gaussian) & 30dB(Gaussian) & 20dB(Poisson) & 30dB(Poisson) \\
\hline \multirow{3}{*}{ EKF } & Normal & $61.2 \pm 33.0$ & $71.5 \pm 23.9$ & $78.0 \pm 31.9$ & $79.5 \pm 23.6$ \\
& Hard & $95.8 \pm 9.5$ & $103.2 \pm 4.5$ & $91.9 \pm 9.1$ & $99.1 \pm 6.1$ \\
& Soft & $42.3 \pm 18.0$ & $47.3 \pm 5.9$ & $62.4 \pm 27.8$ & $57.3 \pm 22.8$ \\
\hline \multirow{3}{*}{$\mathcal{H}_{\infty}$} & Normal & $78.8 \pm 21.6$ & $78.0 \pm 17.3$ & $77.1 \pm 17.6$ & $77.5 \pm 14.1$ \\
& Hard & $104.5 \pm 6.7$ & $104.3 \pm 4.7$ & $104.5 \pm 4.7$ & $104.8 \pm 4.6$ \\
& Soft & $55.0 \pm 7.8$ & $55.2 \pm 7.8$ & $55.2 \pm 7.7$ & $53.2 \pm 6.3$ \\
\hline \hline
\end{tabular}

Similarly, the system measurement equation can be expressed as:

$$
y(t)=D x(t)+e(t)
$$

where $y(t)$ is the measurement vector, $D$ is the measurement matrix that defines the specific input variables, and $e(t)$ is the measurement noise.

$\mathcal{H}_{\infty}$ State-Filter. Considering a system given by Equations (3) and (41), the $\mathcal{H}_{\infty}$ state-filtering problem is to search the optimal estimates of $x(t)$ which satisfy the following performance measure:

$$
\|x(t)-\hat{x}(t)\|_{Q}^{2}<\gamma^{2}\left\{\|v(t)\|_{W^{-1}}^{2}+\|e\|_{V^{-1}}^{2}+\|x(0)-\hat{x}(0)\|_{P(0)^{-1}}^{2}\right\}
$$

where the notation $\|z\|_{G}^{2}$ is defined as the square of the weighted $L_{2}$ norm of $z$, i.e. $\|z\|_{G}^{2}=z^{T} G z$. $P(0), Q, W$, and $V$ are positive definite weighting matrices which are related to confidence measures and chosen by the designers to obtain desired trade-offs. $\hat{x}(0)$ is a priori estimate of $x(0)$, and $\gamma$ is a positive constant that represents a prescribed level of noise attenuation.

The $\mathcal{H}_{\infty}$ estimation for the system described by Equations (3) and (4) with performance criterion (5) consists of the following iterative procedures [10]:

$$
\begin{aligned}
L(t) & =\left(I-\gamma^{-2} Q P(t)+D^{T} V^{-1} D P(t)\right)^{-1} \\
K(t) & =A P(t) L(t) D^{T} V^{-1} \\
\hat{x}(t+1) & =A \hat{x}(t)+B w(t)+K(t)(y(t)-D \hat{x}(t)) \\
P(t+1) & =A P(t) L(t) A^{T}+W
\end{aligned}
$$

It is obvious that the above $\mathcal{H}_{\infty}$ process has a Kalman-like structure. The only difference is that, in $\mathcal{H}_{\infty}$ problem, an additional condition (the Riccati matrix $P$ is positive definite) is required for the solution to exist. It should be mentioned that directly solving Riccati equation (9) for the solution $P(t)$ is not trivial due to its nonlinearity. Instead, iterative procedure is adopted to update $R(t)$ and then $P(t)$, with $R(t)$ defined through $P(t)^{-1}=R(t)^{-1}+\gamma^{-2} Q$, and the details are omitted here. 
Table 2. Difference between the EKF $/ \mathcal{H}_{\infty}$ estimated displacements (from the noisecorrupted synthetic data) and the ground truth (the average displacement is 0.6).

\begin{tabular}{|c|c|c|c|}
\hline Method & Input Data & Maximum Error & Standard Deviation \\
\hline \multirow{3}{*}{ EKF } & $20 \mathrm{~dB}$ (Gaussian) & 0.0246 & 0.0068 \\
& $30 \mathrm{~dB}$ (Gaussian) & 0.0229 & 0.0051 \\
& $20 \mathrm{~dB}$ (Poisson) & 0.0412 & 0.0083 \\
& $30 \mathrm{~dB}$ (Poisson) & 0.0311 & 0.0057 \\
\hline \multirow{3}{*}{$\mathcal{H}_{\infty}$} & $20 \mathrm{~dB}$ (Gaussian) & 0.0245 & 0.0061 \\
& $30 \mathrm{~dB}$ (Gaussian) & 0.0234 & 0.0054 \\
& $20 \mathrm{~dB}$ (Poisson) & 0.0244 & 0.0054 \\
& $30 \mathrm{~dB}$ (Poisson) & 0.0234 & 0.0054 \\
\hline \hline
\end{tabular}

The $\mathcal{H}_{\infty}$ filtering can have many solutions corresponding to different $\gamma$ values. It is observed that the smaller the $\gamma$ value, the smaller the estimation error. On the other hand, the Riccati equation has to have a positive definite solution, which implies that

$$
\begin{gathered}
{\left[A\left(R(t)^{-1}+D^{T} V^{-1} D\right)^{-1} A^{T}+W\right]^{-1}-\gamma^{-2} Q>0} \\
\rightarrow \gamma=\xi \max \left\{\operatorname{eig}\left[A\left(R(t)^{-1}+D^{T} V^{-1} D\right)^{-1} A^{T}+W\right]\right\}^{0.5}
\end{gathered}
$$

where $\max \{\operatorname{eig}(A)\}$ denotes the maximum eigenvalue of the matrix $A$, and $\xi$ is a constant larger than 1 to ensure that $\gamma$ is always greater than the optimal performance level. $\gamma$ value too close to the optimal performance level, i.e. $\xi \approx 1$, might actually lead to numerical instability because of matrix singularity.

\subsection{Elasticity Estimation: $\mathcal{H}_{\infty}$ Parameter Filter}

Parameter State Space Representation. In order to apply the elasticity parameter identification algorithm, the system equation (2) needs be reformulated in the form of $\dot{x}(t)=\mathcal{A}_{c}(x(t)) \theta+\mathcal{B}_{c}$ to facilitate the process. Please note here $x(t)$ is the estimation result from the motion estimation step.

According to the finite element method, the global stiffness matrix $K$ is assembled from the element stiffness $K_{e}$ :

$$
K=\sum K_{e}=\sum \int_{\Omega_{e}} B_{e}^{T} D_{e} B_{e} d \Omega_{e}
$$

where $\Omega_{e}$ is the domain of an arbitrary element $e, B_{e}$ is the local element straindisplacement matrix, and $D_{e}$ is the element material matrix. The element stiffness matrix $K_{e}$ can be stated in terms of its unknown Young's modulus $E_{e}$ :

$$
K_{e}=E_{e} \int_{\Omega_{e}} B_{e}^{T} D_{e}{ }^{\prime} B_{e} d \Omega_{e}=E_{e} K_{e}{ }^{\prime}
$$

With $U$ and $\dot{U}$ the estimation results from the $\mathcal{H}_{\infty}$ state-filter, we can iteratively recast $K U$ to $G_{1} E$ (and $K \dot{U}$ to $\left.G_{2} E\right) L^{2}$.

\footnotetext{
${ }^{2}$ The detailed converting procedures can be found in [12]
} 

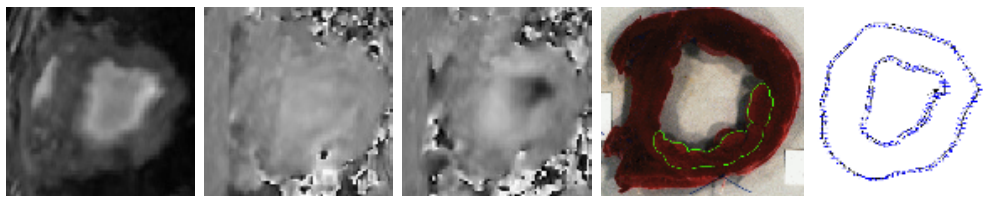

Fig. 3. From left to right: Canine MR magnitude image, x-direction, y-direction phase contrast velocity image, TTC stained postmortem myocardium with the infracted tissue highlighted, displacement constraints on the endo- and epi-cardial boundaries throughout the cardiac cycle, and blowup view of one point's trajectory.

Submitting $C=\alpha M+\beta K, K U=G_{1} E$, and $K \dot{U}=G_{2} E$ into Equation 2] we now have the following identification form of the system equation:

$$
\dot{x}(t)=F x+\mathcal{A}_{c}(x(t)) \theta+B_{c} w(t)
$$

where

$$
\begin{array}{cc}
\theta=E, \quad x(t)=\left[\begin{array}{c}
U(t) \\
\dot{U}(t)
\end{array}\right], \quad w(t)=\left[\begin{array}{l}
0 \\
R
\end{array}\right], & 0 \\
\mathcal{A}_{c}=\left[\begin{array}{cc}
0 & 0 \\
-M^{-1} G_{1} & -\beta M^{-1} G_{2}
\end{array}\right], \quad B_{c}=\left[\begin{array}{cc}
0 & 0 \\
0 & M^{-1}
\end{array}\right], \quad F=\left[\begin{array}{cc}
0 & I \\
0 & -\alpha I
\end{array}\right]
\end{array}
$$

Assuming that the elasticity parameter vector $\theta$ is temporally constant, we have the following augmented system:

$$
\left[\begin{array}{c}
x \dot{(t}() \\
\dot{\theta}
\end{array}\right]=\left[\begin{array}{c}
F x \\
0
\end{array}\right]+\left[\begin{array}{c}
\mathcal{A}_{c}(x) \theta+B_{c} w(t) \\
0
\end{array}\right]+\left[\begin{array}{c}
v_{p}(t) \\
0
\end{array}\right]
$$

and the measurement equation is:

$$
y(t)=\left[\begin{array}{ll}
D & 0
\end{array}\right]\left[\begin{array}{l}
x \\
\theta
\end{array}\right]+e(t)=H\left[\begin{array}{l}
x \\
\theta
\end{array}\right]+e(t)
$$

$\mathcal{H}_{\infty}$ Parameter-Filter. To solve the elasticity estimation problem, we introduce another cost function which seeks to achieve a guaranteed disturbance attenuation level $\gamma_{p}$ between the unknown quantities and the parameter estimate error of the form:

$$
\|\theta-\hat{\theta}\|_{Q_{p}}^{2}<\gamma_{p}^{2}\left\{\left\|v_{p}\right\|^{2}+\|e\|^{2}+|\theta-\hat{\theta}(0)|_{Q_{p 0}}^{2}+\left|x(0)-\hat{x}_{p}(0)\right|_{Q_{p 1}}^{2}\right\}
$$

Here, $\hat{x}_{p}(0)$ is the initial estimate, and $Q_{p}, Q_{p 0}, Q_{p 1}$ are the weighting factors. Under this criterion, the $\mathcal{H}_{\infty}$ algorithm for the system (13) and (14) is described for $\gamma_{p}>\gamma^{*}$, with $\gamma^{*}$ is the best-achievable disturbance attenuation level:

$$
\left[\begin{array}{c}
\hat{\dot{x}} \\
\hat{\dot{\theta}}
\end{array}\right]=\left[\begin{array}{cc}
F & \mathcal{A}_{c}(x) \\
0 & 0
\end{array}\right]\left[\begin{array}{c}
\hat{x} \\
\hat{\theta}
\end{array}\right]+\left[\begin{array}{c}
B_{c} w(t) \\
0
\end{array}\right]+\Sigma^{-1} H^{T}(y-D \hat{x})
$$



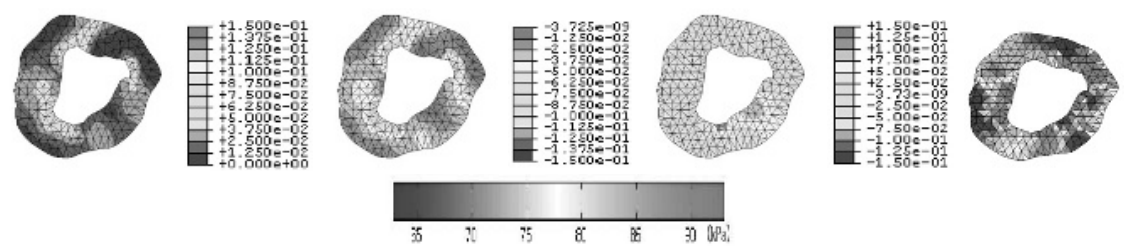

Fig. 4. From left to right: estimated radial (R) strain, circumferential (C) strain, R$\mathrm{C}$ shear strain maps between end-diastole (ED) and end-systole(ES), and estimated Young's modulus distribution (the color scale is on the bottom).

where $\Sigma$ satisfies the Riccati equation with initial condition $\Sigma(0)=$ $\operatorname{diag}\left(Q_{p 1}, Q_{p 0}\right)$ :

$$
\dot{\Sigma}=-\Sigma\left[\begin{array}{cc}
F & \mathcal{A}_{c}(x) \\
0 & 0
\end{array}\right]-\left[\begin{array}{cc}
F^{T} & 0 \\
\mathcal{A}_{c}^{T} & 0
\end{array}\right] \Sigma+\left[\begin{array}{cc}
D^{T} D & 0 \\
0 & -\gamma_{p}^{-2} Q_{p}
\end{array}\right]-\Sigma\left[\begin{array}{ll}
I & 0 \\
0 & 0
\end{array}\right] \Sigma
$$

Some of the related theoretical and practical discussions can be found in 212 .

\section{Results and Discussions}

\subsection{Validation with Synthetic Data}

The iterative sequential $\mathcal{H}_{\infty}$ estimator is validated with computer-synthesized data of an object undergoing deformation in the vertical direction with fixing the bottom side. As shown in Fig. 1, the object has unit thickness, and the material properties and other parameters are taken as $E_{\text {hard }}=105$ for the hard (red) part, $E_{\text {normal }}=75$ for the normal (white) part, and $E_{\text {soft }}=45$ for the soft (blue) part, $\mathrm{D}=16, \mathrm{~L}=28$, Poisson's ratio $\nu=0.49$. Under this exact model and boundary conditions, we get displacements for all nodal points, which are labelled as the ground truth data set. Then only a subset of the displacements are selected as the partial measurements on the kinematics, as shown in Fig. 1 where only displacements of the yellow points are known. Finally, different types (Gaussian and Poisson) and levels (20dB and $30 \mathrm{~dB})$ of noises are added to these displacements to generate the noisy data. For comparison purpose, the extended Kalman filter (EKF) framework of [1] is used to recover the displacement and elasticity modulus distribution as well.

Quantitative assessments and comparisons of the $\mathcal{H}_{\infty}$ and EKF results are presented in Table 1, and the recovered elasticity distributions are shown in Fig. 2 In order to validate the motion estimate results, point-by-point positional errors between the algorithm and the ground truth data are computed as shown in Table 2. Overall, the EKF results for Poisson-corrupted input data are not very satisfactory, which indicate that if the assumptions on the noise statistics are violated, it is possible that small noise errors may lead to large estimation errors for EKF. On the other hand, all the tables and figures illustrate that very similar results are obtained using the iterative sequential $\mathcal{H}_{\infty}$ framework for two sets of data contaminated by different types of noise, showing its desired robustness for real-world problems. 


\subsection{Canine Image Application}

Fig. 3 demonstrates the MR phase contrast image data set. The regional volume of the postmortem injury zone is found by digitizing color photographs of the triphenyl tetrazolium chloride (TTC) stained post mortem myocardium (Fig. 3), which provides the clinical gold standard for the assessment of the image analysis results.

Myocardial boundaries and frame-to-frame boundary displacements are extracted using a unified active region model strategy 13 (as shown in Fig. 3). The estimated radial (R), circumferential $(\mathrm{C})$, and $\mathrm{R}-\mathrm{C}$ shear strain maps, and the material elasticity distribution are shown in Fig. 4 , with initial Young's modulus set to 75000 Pascal. These maps exhibit vastly different motion and material parameters at the infarct zone from the normal tissues, and the patterns are in good agreement with the highlighted histological results of TTC stained postmortem myocardium (Fig. 3). Further, the infarct zone myocardial tissues are relatively stiffer than normal with larger Young's modulus values, which has been observed experimentally earlier.

Acknowledgments. Thanks to Dr. Albert Sinusas of Yale University for the canine experiments and the imaging data. This work is supported by HKRGCCERG HKUST6151/03E and National Basic Research Program of China (No: 2003CB716104).

\section{References}

1. L.L. Creswell, M.J. Moulton, S.G. Wyers, J.S. Pirolo, D.S. Fishman, W.H. Perman, K.W. Myers, R.L. Actis, M.W. Vannier, B.A. Szabo, and M.K. Pasque. An experimental method for evaluating constitutive models of myocardium in in vivo hearts. American Journal of Physiology, 267:H853-H863, 1994.

2. G. Didinsky, Z. Pan, and T Basar. Parameter identification for uncertain plants using $\mathcal{H}_{\infty}$ methods. Automatica, 31(9):1227-1250, 1995.

3. A.J. Frangi, W.J. Niessen, and M.A. Viergever. Three-dimensional modeling for functional analysis of cardiac images: A review. IEEE Transactions on Medical Imaging, 20(1):2-25, 2001.

4. Z. Hu, D. Metaxas, and L. Axel. In-vivo strain and stress estimation of the heart left and right ventricles from mri images. Medical Image Analysis, 7(4):435-444, 2003.

5. W.S. Kerwin and J.L. Prince. The kriging update model and recursive space-time function estimation. IEEE Transactions on Image Processing, 47(11):2942-2952, 1999.

6. H. Liu and P. Shi. Simultaneous estimation of left ventricular motion and material properties with maximum a posteriori strategy. In IEEE Computer Vision and Pattern Recognition, pages 161-169, 2003.

7. E.W.B. Lo, H. Liu, and P. Shi. $H_{\infty}$ filtering and physical modeling for robust kinematics estimation. In IEEE International Conference on Image Processing, volume 2, pages 169-172, 2003. 
8. F.G. Meyer, R.T. Constable, A.J. Sinusas, and J.S. Duncan. Tracking myocardial deformation using spatially constrained velocities. IEEE Transactions on Medical Imaging, 15(4):453-465, 1996.

9. R. Muthupilla, D.J. Lomas, P.J. Rossman, J.F. Greenleaf, A. Manduca, and R.L. Ehman. Magnetic resonance elastography by direct visualization of propagating acoustic strain waves. Science, 269(5232):1854-1857, 1995.

10. X. Shen and L. Deng. A dynamic system approach to speech enhancement using the $\mathcal{H}_{\infty}$ filtering algorithm. IEEE Transactions on Speech and Audio Processing, 7(4):391-399, 1999.

11. P. Shi and H. Liu. Stochastic finite element framework for simultaneous estimation of cardiac kinematics functions and material parameters. Medical Image Analysis, 7(4):445-464, 2003.

12. P. Shi, H. Liu, and A. Sinusas. Robust filtering strategies for soft tissue Young's modulus characterization. In IEEE International Symposium on Biomedical Imaging: Macro to Nano, in press, 2004.

13. A.L.N. Wong, H. Liu, A. Sinusas, and P. Shi. Spatiotemporal active region model for simultaneous segmentation and motion estimation of the whole heart. In IEEE Workshop on Variational, Geometric and Level Set Methods in Computer Vision, pages 193-200, 2003.

14. Y. Zhu and N.J. Pelc. A spatiotemporal model of cyclic kinematics and its application to analyzing nonrigid motion with $\mathrm{mr}$ velocity images. IEEE Transactions on Medical Imaging, 18(7):557-569, 1999. 